\title{
Estrategias resilientes ante la situación adversa de los desahucios
}

\section{Strategies for resilience in adverse situations involving evictions}

\author{
María de las Olas Palma García \\ Universidad de Málaga, España \\ mpalma@uma.es \\ Ana Cristina Ruiz Mosquera \\ Universidad de Málaga, España \\ acristinaruizmosquera@gmail.com
}

Recibido: 30/08/2015

Revisado: 01/10/2015

Aceptado: 09/03/2016

Disponible on line: 01/07/2016

\section{Resumen}

Los desahucios en España se han convertido en los últimos años en un fenómeno social emergente, de interés tanto para la investigación como para la intervención de los profesionales de la acción social. Aun así, no existen estudios suficientes relacionados con esta situación adversa, y menos aún centrados en las respuestas resilientes que las personas son capaces de ofrecer ante ella. Con el objetivo de identificar los factores internos y externos que se presentan en la experiencia de las familias que viven procesos de desahucios y que les están permitiendo desarrollar estrategias resilientes ante dicha adversidad, se lleva a cabo la presente investigación en la que participan 20 sujetos, pertenecientes a familias que acuden a la Plataforma de Afectados por las Hipotecas (PAH) de Málaga. A los participantes se les realiza una entrevista semi-estructurada y a través de ellas se han podido definir las características socio-familiares de las personas afectadas, las diferentes estrategias de afrontamiento desarrolladas, las principales fuentes de apoyo con las que han contado, así como las preocupaciones que les han acompañado. Los resultados muestran los principales factores internos y externos que están presenten en las estrategias resilientes que han desarrollado las familias afectadas por los desahucios durante el proceso vivido y destacan como fuente de apoyo informacional a la PAH. Se concluye con la necesidad de continuar con esta línea de investigación para tratar de diseñar intervenciones que refuercen y fomenten las estrategias de afrontamiento ante la situación adversa del desahucio.

Palabras clave: resiliencia, desahucios, adversidad, intervención social, afrontamiento.

\begin{abstract}
In recent years in Spain, evictions have become an emerging social phenomenon, of interest for both research and intervention by social action professionals. However, there are insufficient studies related to this adverse situation, particularly concerning the resilient responses that people are able to offer when faced with it. This research was carried out in order to identify the internal and external factors present in the experience of families who experience eviction processes and that are enabling them to develop resilient strategies in the face of such adversity. The research involved the participation of 20 subjects, belonging to families forming part of the Platform for Mortgage Victims (Plataforma de Afectados por las Hipotecas, or PAH) in Malaga. The participants underwent a semi-structured interview, which permitted the definition of the socio-family characteristics of the affected persons, the different coping strategies they developed, the main sources of support on which they relied, as well as the concerns they have had. The results show the main internal and external factors that are present in the resilient strategies developed by families in the course of the evictions they have experienced and highlight the PAH as a source of informational support. The conclusion is that there is a need to continue with this line of research to seek to design interventions that reinforce and encourage coping strategies for adverse situations involving eviction.
\end{abstract}

Keywords: resilience, evictions, adversity, social intervention, coping.

Referencia normalizada: Palma García, Ma O. y Ruiz Mosquera, A.C. (2016): «Estrategias resilientes ante la situación adversa de los desahucios». Cuadernos de Trabajo Social, 29(2): 299-308.

Sumario: Introducción. 1. Metodología. 2. Resultados. 3. Conclusiones. 4. Referencias bibliográficas. 


\section{Introducción}

\section{El desahucio como situación adversa}

La realidad de los desahucios no es un acontecimiento nuevo pero sí actual, dado el incremento de casos sobrevenidos en los últimos años como consecuencia de la crisis económica que atraviesa nuestro país.

El concepto de desahucio o, también conocido como lanzamiento judicial, hace referencia a la ejecución forzosa instada por un juzgado para que una familia abandone su vivienda ante la imposibilidad de hacer frente al pago de la hipoteca o alquiler. Con ello se describe una situación adversa, en la que la amenaza de privación de vivienda para muchas familias puede suponer el inicio de un camino hacia la vulnerabilidad (Navarro, 2005). Es así como las familias que sufren el desalojo del bien inmueble en el que habitaban en favor de su propietario legítimo se sitúan a solo un paso del camino a la exclusión social, ya que la ausencia o precariedad en la vivienda, «representa la cristalización de la vulnerabilidad social» (Malagón, 2008, p. 101).

La Declaración Universal de los Derechos Humanos incluye la vivienda dentro de los derechos necesarios para poder tener un nivel de vida adecuado. Es algo más que un espacio físico: es un recurso fundamental para el desarrollo de la vida en sociedad, un factor clave de integración social, de formación de hogares, de emancipación y de participación de una persona en su entorno. De hecho, de acuerdo con Cortés (2000) «disponer de una vivienda en nuestros días, no sólo es un derecho sino que se ha convertido en una necesidad, es un bien necesario» (p. 298) asociado, a su vez, a la posibilidad de realización personal.

Este enfoque es el que recuerda la Fundación FOESSA (2013) en su informe «La vivienda en España en el siglo XXI» en el que considera

La vivienda como un bien para la satisfacción de una necesidad, y no la consideración de la vivienda como un objeto de inversión para el beneficio, ya que el alojamiento de la población en condiciones adecuadas es un satisfactor que garantiza la necesidad básica de subsistencia/salud. La vivienda es un espacio vital, imprescindible para la socialización, para ser parte de una sociedad, es decir, es un factor de inclusión social de primer orden (p. 4).
Sin embargo, en el contexto reciente de la política de vivienda en la sociedad española, esta necesidad ha estado sometida a los intereses especulativos que ocasionaron el auge y estallido de la «burbuja inmobiliaria» y con ello la pérdida de seguridades irreales hacia la vivienda en las que las familias creían estar. Fruto de esta situación, nos encontramos ante una realidad clara de adversidad en la que se han visto inmersas todas aquellas personas afectadas por los desahucios.

En cifras globales, según el informe «Efectos de la crisis económica en los órganos judiciales», publicado por el Consejo General del Poder Judicial (2015), los lanzamientos judiciales practicados en 2014 fueron 68.091, el 1,3 por ciento superior a los ejecutados en el año anterior. De ellos, el 52,9 por ciento fueron consecuencia de procedimientos derivados de la Ley de Arrendamientos Urbanos y el 42,2 por ciento, de ejecuciones hipotecarias. En paralelo, no existen indicadores significativos de que la mejora de la economía incida a corto-medio plazo en la reducción de la elevada tasa de paro existente, por lo que se prevé continúe en muchas familias la dificultad sobrevenida de poder atender sus hipotecas o arrendamientos. De acuerdo con Sabater y Giró (2015) la vinculación entre crisis financiera $\mathrm{y}$ desahucio se establece a partir de factores que, bien en sí mismos, o bien en combinación (el paro y la deuda hipotecaria), precipitan el proceso de empobrecimiento. Estos autores relacionan la situación de las personas con desahucios como consecuencia de la crisis económica porque han perdido su empleo, han visto disminuir su salario, se encuentran inmersos en recortes, expedientes de regulación de empleo (ERE) o reducciones de horario, dependen de ayudas públicas cada vez más menguadas, se han encontrado con nuevas situaciones biográficas (enfermedad, dependencia, divorcio, etc.) que han incidido en la disminución de su nivel de vida hasta no poder afrontar sus gastos básicos. Entre estos gastos, la vivienda representa la mayor inversión, fundamentalmente por los contratos hipotecarios que sujetan a personas y familias a un gasto permanente a lo largo de su vida. Además del desempleo, la literatura relacionada aporta otros elementos que caracterizan la situación de adversidad que viven estas personas.

Las consecuencias derivadas de la situación personal y social en la que se ven inmersas las 
familias en procesos de desahucios se pueden considerar, de acuerdo con Terris (1992) un problema de salud pública. En estas situaciones los afectados no pueden mantener un nivel de vida adecuado debido a las consecuencias tanto psicológicas como físicas que este proceso tiene sobre la salud. Según un estudio de la Escuela Andaluza de Salud Pública (EASP, 2014) elaborado en colaboración con la Universidad de Granada y la Plataforma Stop Desahucios, la mayoría de los afectados por el problema de las hipotecas sufren niveles graves de ansiedad y depresión. El proceso de desahucio puede durar varios años, comenzando con un cambio en la situación personal que suele ser la pérdida del empleo o reducción considerable del salario que implica vivir acompañado de estrés negativo fruto de la amenaza constante de poder perder tu vivienda (EASP, 2014). Junto a los efectos sobre la salud mental, este mismo estudio evidencia el deterioro producido en la salud física durante el proceso de desahucio, al incidir de forma directa en los hábitos de salud, tales como horas de sueño, dieta, actividad física e incremento en el consumo de medicamentos y uso de servicios sanitarios. El riesgo de suicidio es otra de las posibles consecuencias derivadas, según dicho estudio un tercio de los encuestados presenta un nivel alto o moderado. Otro de los aspectos analizado en estudios previos es el relacionado con el apoyo percibido por las personas que experimentan un proceso de desahucio. Las principales fuentes de apoyo provienen de los familiares y amigos, siendo las instituciones públicas las que se muestran menos presentes en estos procesos (Arredondo y Palma, 2013).

Dejar de tener casa es una de las razones por las que la ONG Save The Children asegura que la infancia en España es uno de los sectores más afectados por la crisis. En la literatura revisada se constata la presencia de menores en las familias afectadas por desahucios (p. e. Arredondo y Palma, 2013; Valiño, 2013). A estos menores, el tener que abandonar sus hogares les afecta de manera grave en sus derechos fundamentales de educación, salud y protección. No se ha investigado lo suficiente sobre qué otras consecuencias directas tiene para estos menores la experiencia de un desahucio, aunque no es difícil imaginar su especial vulnerabilidad ante esta situación.

En esta realidad, como respuesta social a los desahucios, surgen las plataformas de afectados por las hipotecas $(\mathrm{PAH})$. La primera se crea en Barcelona, en 2008, con el propósito de agrupar a personas con dificultades para pagar la hipoteca o que se encuentran en proceso de ejecución hipotecaria y personas que con su labor pudieran ofrecer su ayuda de forma altruista. Frente a una legislación hipotecaria que condena a las personas sobre-endeudadas a la exclusión social, la PAH se constituye como un lugar de encuentro y empoderamiento (Valiño, 2013). En concreto, la PAH orienta su lucha a conseguir un cambio normativo que posibilite una segunda oportunidad a las personas afectadas por la hipoteca, tal y como sucede en el resto de países del entorno. Mientras este cambio normativo no se materializa, la PAH a través de la campaña Stop Desahucios y de la tarea de su obra social evita desalojos y acompaña el realojamiento adecuado de las familias.

\section{La resiliencia como estrategia ante los desa- hucios}

La resiliencia es un concepto de interés en la actualidad por la importancia que tiene para la promoción del desarrollo (Salgado, 2005), definido como la capacidad de las personas para desarrollarse y crecer psicológicamente sanas y exitosas, a pesar de estar expuestas a situaciones adversas que amenazan su integridad (Rutter, 1993).

Las condiciones adversas que pueden relacionarse con la resiliencia son múltiples. La mayoría de los estudios realizados hasta el momento se han centrado en situaciones relacionadas con la pobreza, el abuso parental, la discriminación racial, la discapacidad, entre otras, aunque el valor de este nuevo paradigma ofrece un potencial interés ante todo tipo de situaciones adversas a las que se enfrentan las personas a lo largo de su vida, también los desahucios.

La capacidad de respuesta ante una adversidad es siempre un juego dinámico entre recursos, fortalezas y debilidades, que puede desarrollarse durante toda la vida y debe ser analizado en cada caso en particular (Gauto de Paz, 2007). La conducta resiliente, por tanto, se explica más como una particular combinación de factores resilientes que como la suma de los mismos (Osborn, 1994). Entre dichos factores, la literatura en torno a resiliencia ha venido aportando evidencias respecto a aquellos internos a las personas, que les permiten poner en marcha estrategias resilientes ante las situaciones adversas con las que conviven, $\mathrm{y}$ aquellos otros externos a 
ellas, en los que se apoyan para articular dicha respuesta.

En relación a los factores internos, según Mrazek y Mrazek (1987) la persona resiliente es aquella que reconoce las situaciones que le suponen un peligro, por lo tanto trata de dar una respuesta rápida ante la situación amenazante. Tienen la habilidad de asumir la responsabilidad propia cuando se toman decisiones incluso si la decisión tiene algún tipo de riesgo. Buscan información, se preocupan de aprender todo lo relacionado con el entorno que les rodea. La persona resiliente tiende a desarrollar la capacidad de hacerse cargo de sí mismo, trata de alejar los sentimientos intensos, creando así una desvinculación afectiva pero manteniendo relaciones que le ayuden a subsistir en momentos críticos. Son capaces de identificar los eventos negativos, y realizar una reconstrucción cognitiva del dolor, aceptando y superándolos. Son además personas capaces de confiar en sí mismas y mostrar iniciativa para emprender acciones o relaciones con otros (Vanistendael y Lecomte, 2002).

Como factores externos que contribuyen a promover la resiliencia, la literatura relacionada ha venido destacando toda una serie de recursos activos presentes en la comunidad que, de acuerdo con Kaztman (1999) ofrecen una estructura de oportunidades ante la adversidad. En este sentido, ante un momento y circunstancia determinada como la situación de desahucio, se activan relaciones interpersonales de protección, relaciones de apoyos que surgen de la movilización de la comunidad, de su organización y participación comunitaria que constituyen un capital social clave para el proceso de recuperación y fortalecimiento (Coloma, 2009).

Bajo este enfoque, la finalidad de este estudio se dirige a identificar los factores internos y externos presentes en la experiencia de las familias que viven procesos de desahucios que les están permitiendo desarrollar estrategias resilientes ante dicha adversidad. Para la investigación e intervención social, el enfoque de la resiliencia muestra un futuro prometedor y desafiante que ha de ser explorado en el contexto adverso y de emergencia social que suponen los desahucios.

\section{Metodología}

La presente investigación se realiza desde un enfoque cualitativo con el propósito de conocer las estrategias resilientes puestas en marcha en las familias en procesos de desahucios a través de la experiencia relatada por ellas mismas. Para ello se lleva a cabo un estudio descriptivo y transversal, que persigue obtener conocimientos en el campo de la resiliencia con el fin de aplicarlos de forma concreta a la realidad de los desahucios.

\subsection{Participantes}

Para la presente investigación se ha llevado a cabo un muestreo de tipo intencional buscando de forma deliberada la representatividad del colectivo de personas afectadas por los desahucios de sus viviendas habituales en los últimos años, entre quienes participan de la Plataforma de Afectados por los Desahucios de Málaga. Se ha identificado un total de 20 participantes, en su mayoría hombres, con un rango de edad de 26 a 64 años, preferentemente de nacionalidad española y sin hijos a cargo (véase Cuadro 1).

\subsection{Instrumentos}

El instrumento utilizado para la recogida de datos ha sido la entrevista semiestructurada. Se ha diseñado de forma específica para este estudio, compuesta por 10 ítems de carácter abierto de los que se han obtenido datos relativos a la com-

\begin{tabular}{|c|c|c|c|c|}
\hline $\begin{array}{c}\mathbf{N}^{\circ} \\
\text { entrevistado }\end{array}$ & Sexo & Edad & Nacionalidad & $\begin{array}{c}\text { Hijos } \\
\text { a cargo }\end{array}$ \\
\hline E1 & Mujer & 52 & Española & Sí \\
E2 & Hombre & 43 & Española & Sí \\
E3 & Mujer & 40 & Española & Sí \\
E4 & Hombre & 57 & Española & No \\
E5 & Hombre & 43 & Chilena & Sí \\
E6 & Hombre & 51 & Marroquí & Sí \\
E7 & Hombre & 54 & Marroquí & No \\
E8 & Mujer & 36 & Española & No \\
E9 & Hombre & 40 & Argentina & No \\
E10 & Hombre & 33 & Española & No \\
E11 & Mujer & 29 & Española & No \\
E12 & Hombre & 64 & Española & No \\
E13 & Mujer & 42 & Española & No \\
E14 & Hombre & 26 & Española & No \\
E15 & Hombre & 63 & Española & No \\
E16 & Mujer & 52 & Española & No \\
E17 & Hombre & 49 & Española & No \\
E18 & Hombre & 35 & Española & Sí \\
E19 & Hombre & 55 & Española & No \\
E20 & Hombre & 38 & Española & Sí \\
\hline
\end{tabular}

Cuadro 1. Características sociofamiliares de los participantes. 
posición de la familia, preocupaciones sobre el proceso de desahucio, principales fuentes de apoyo durante el proceso y de afrontamiento personal del problema haciendo referencia al cómo $\mathrm{y}$ al dónde actuaron para encontrar la salida a la situación adversa. Con el fin de poder realizar comparaciones y posibles contrastes entre las entrevistas que permita organizar conceptualmente los datos y presentar la información siguiendo un patrón o regularidad emergente, se han establecido cuatro categorías para su análisis de contenido: (1) Preocupaciones ante la situación adversa; (2) Fortalezas personales ante la adversidad del desahucio; (3) Fuentes de apoyo externo presentes en el proceso de desahucio; y (4) Visión de futuro como elemento facilitador.

\subsection{Procedimiento}

Las entrevistas se llevaron a cabo durante el mes de abril de 2015. El lugar elegido para su realización fue la sede de la Plataforma de Afectados por las Hipotecas de Málaga y la duración media de las mismas ha sido de una hora. En todos los casos, tras invitarlos a participar en la investigación se ha informado sobre la voluntariedad y finalidad de la entrevista y confidencialidad de los datos recabados.

El tratamiento de los datos se ha llevado a cabo mediante el análisis de contenido. Las entrevistas han sido transcritas y posteriormente procesadas para eliminar referencias a datos personales y dotarlas de garantía de confidencialidad. Tras su lectura inicial, se ha realizado el análisis de contenido, identificando la frecuencia de aparición de las categorías a observar y llevando a cabo su posterior tratamiento cualitativo.

\section{Resultados}

En una primera aproximación a las características sociofamiliares de los participantes, se observa que todos ellos formalizaron los préstamos hipotecarios de sus viviendas con anterioridad al año 2005, fecha previa a la crisis económica que atraviesa la sociedad española. En dicho momento, creían tener una situación económica estable y una seguridad ante su vivienda, que posteriormente derivó en desempleo o en contrataciones precarias que les imposibilitaba seguir haciendo frente a los pagos de vivienda.

En relación a su composición familiar, los resultados muestran que las familias entrevistadas están compuestas en su mayoría por matrimo- nios de edad avanzada con hijos ya independientes y nietos, que viven situaciones de desahucios a consecuencia de haber apoyado económicamente a éstos. Les siguen otros que viven solos y por último, parejas con hijos mejores a cargo (5 de las 20 familias con dos menores a cargo de edades inferiores a los 9 años).

A continuación se muestran los resultados obtenidos en función de las categorías de análisis previstas en el diseño.

\subsection{Preocupación ante la situación adversa}

Todos los participantes reconocen haber sentido en un primer momento miedo, ansiedad y haberse visto envueltos en un proceso de depresión ante la experiencia en la que se encontraban inmersos. Expresiones tales como: «Se me vino el mundo abajo» (E13); «No encontraba salida posible» (E20); «No servía para nada, fracasé» (E1), hacen referencia al duro trance que genera en una persona verse afectada por un proceso de desahucio. Sus mayores y primeras preocupaciones eran no poder hacer frente a los pagos y, consecuentemente perder la vivienda por la que tanto había luchado y que tanto deseaban.

Me quedé varias noches en vela, pese al esfuerzo económico y privaciones materiales no podíamos seguir pagando la hipoteca, no me salían las cuentas, definitivamente nos íbamos a tener que ir de la casa (E18).

No se trata de perder un inmueble como algo material sino «su vivienda», donde habían vivido tantos momentos y que tantos sentimientos les suscitaba.

Mis hijos han crecido en esta casa, he pasado momentos tan felices en ella que no puedo imaginarme tener que irme (E6).

Al principio lloraba por los rincones, no me lo podía creer, la que había sido la sede de nuestros momentos felices tenía fecha de caducidad (E9).

Otra preocupación de los participantes ante la idea del desahucio es la importancia de la imagen social. Es decir, la importancia que para algunas personas tiene el qué dirán. Se perciben a sí mismos como perdedores, al inicio la situación adversa les provoca un sentimiento de frustración social, perciben un deterioro de la imagen que su entorno pueda tener sobre ellos. Relatos como los siguientes ponen esta preocupación de manifiesto: 
Desde que nos casamos vivimos en este barrio, tenemos muy buena relación con todos y no sabía cómo decirles que nos vamos a tener que ir, me daba vergüenza contar la situación por la que estábamos pasando (E4).

Me iba a tener que ir del barrio con una mano delante y otra detrás, todo por haber tenido la torpeza de no administrarme bien y contratar una hipoteca que a día de hoy no puedo pagar (E13).

Lo único que no quería era que mi padre se enterara, ¡qué iba a pensar! Todos mis hermanos con sus casas y tan felices y ahora uno de sus hijos desahuciado (E17).

En relación a otras preocupaciones, se observa en los participantes el temor común a no poder cubrir la necesidad de comer por invertir los ingresos de los que disponían al pago de la hipoteca, lo que empuja a elegir entre seguir pagando la hipoteca o pasar necesidades alimenticias: «Preferíamos seguir pagando al banco y no comer antes que perder nuestra casa» (E19).

Las preocupaciones señaladas por los participantes repercuten de forma directa en sus estados de salud. Destaca además cómo estas situaciones de precariedad económica están afectando a su vez a familias especialmente vulnerables como personas con enfermedades crónicas, mayores y menores.

Ahora que lo pienso por lo que más me preocupé fue por mi salud, caí en una depresión y comencé a auto-medicarme. Pasaba el día durmiendo. No sé si sería la medicación pero estaba siempre cansada y sólo tenía ganas de llorar. Dejé incluso de comer (E16).

Hace unos años me detectaron que soy diabético, por lo que tengo que cuidar mucho lo que como pero con esta situación me estoy descontrolando demasiado (E7).

Mi preocupación era la salud de mi madre. Ella fue quien me avaló la hipoteca (E10).

Del análisis de las entrevistas se desprende el valor que persiste en la sociedad sobre la importancia y seguridad de tener una vivienda en propiedad, tanto por parte de los afectados por los desahucios como de sus familiares y conocidos.

Nadie quiere vivir pagando algo que no va a ser suyo, vas pagando poco a poco una casa que poder dejarle a tus hijos (E3).

Mi madre siempre me decía: tú tienes que tener tu casita y a partir de ahí formar tu familia; supongo que será lo que piensan todas las madres (E10).

\subsection{Fortalezas personales ante la adversidad del desahucio}

Los resultados muestran que todos los entrevistados han contado con factores personales resilientes en algún momento del proceso vivido ante el desahucio, que han conocido a partir de su convivencia con la adversidad.

Fui capaz de hacerle frente a la situación. Crees que nunca te va a tocar a ti, pero cuando te ocurre te haces experto y se pulsa el botoncito que activa en tu cabeza el interés en conocer cómo puedes solucionarlo (E12).

Los entrevistados mencionan que en el momento que reciben la primera notificación del banco su estado es de angustia y ansiedad por el problema que se les viene encima, pero también reconocen que su principal herramienta son ellos mismos, como demuestran con sus testimonios

Él (refiriéndose a su actual pareja) fue quién me enseñó que yo era lo suficientemente fuerte como para hacer frente al problema que se me había venido encima (...) a día de hoy me considero autosuficiente (E13).

Cerraba los ojos y me quedaba sólo conmigo mismo. Yo me decía: sigue que tú puedes con esto y más (E19).

Lloré todas las mañanas durante una semana, escondí la carta para que mi mujer no la viera y evitar que se preocupara. Me iba al banco a ver que podía hacer, y traté de buscar los medios económicos como fuera (E10).

Los afectados pusieron interés en hacer frente a la problemática y reconociendo las dificultades tomaron las riendas de la situación tratando de buscar una salida.

Verse afectado por la hipoteca es una situación en la que te ves de pronto pero que tiene salida, aunque al principio no se la veas (E19).

La mayoría de los participantes reconocen haber salido fortalecidos tras verse afectados por las hipotecas,

«Experiencias como estas te fortalecen en la vida» (E3).

Nunca piensas que te va a tocar a ti pero, a veces en la vida te tienen que pasar desgracias como esta para conocerte realmente y saber hasta dónde eres capaz de llegar (E1).

He salido muy fortalecido de esta experiencia, no te voy a negar que siempre he sido fuerte pero no te conoces bien hasta que las cosas no te pasan y de esta experiencia como de otras se aprende (E12). 
De ahora en adelante voy a ser capaz de afrontar nuevas situaciones teniendo la certeza de que todo tiene alguna salida, aunque no siempre sea del todo favorable (E9).

Otros no reconocen aún sus nuevas fortalezas pero creen que han podido desarrollarlas

No me considero más fuerte, al menos por ahora, pero quizás si en el futuro me viene otro problema - que seguro que vendrá- siempre recordaré estos meses tan malos que estoy pasando y a lo mejor me sirve (E7).

\subsection{Fuentes de apoyo externo presentes en el proceso de desahucio}

Los participantes coinciden en señalar que poseen una red social fuerte que les ha servido: la familia extensa como principales generadores de apoyo, seguidos del apoyo recibido por parte de sus amigos y conocidos. Solo dos de los entrevistados no contaban con este tipo de apoyo debido a que su familia extensa se encuentra en su país de origen: «Desde lejos te apoyan, te invitan a volver a casa con ellos» (E7).

Los resultados muestran que los padres e hijos, y en algunos casos, amigos, de los participantes han sido quienes les han ofrecido principalmente apoyo de tipo emocional a lo largo del proceso.

Sólo con escuchar, tranquilo que estamos aquí contigo, ya te da fuerzas para seguir adelante (E17).

Valoro mucho cada abrazo que he recibido de mis amigos, mucho más que si me hubieran dado dinero porque en esos momentos era lo que más necesitaba, sentir que no estábamos solos (E11).

Sin embargo, el apoyo material reconocen haberlo recibido sólo de padres (la mayoría incluso avalaron la hipoteca de la vivienda) o incluso algunos, señalan no haber recibido ningún tipo de apoyo material durante el proceso.

Lo que más me partía el alma era ver a mi madre llorando; había pedido un préstamo personal para hacer frente a mi hipoteca y, ni aun así conseguíamos seguir adelante (E2).

Mis padres han trabajado toda su vida y no dudaron en apoyarme económicamente, no querían vernos pasar por esta situación, ni que tuviera ningún tipo de repercusión en sus nietos (E20).

No te puedo decir que haya dejado de comer porque el día que no tenía comida me iba a casa de mi madre y nunca me ha faltado (E3).
Por último, el apoyo informacional tiene un nombre para todos los entrevistados: Plataforma de Afectados por la Hipoteca. Todos los participantes hacen especial hincapié en la información recibida por parte de la plataforma

Nadie te puede informar mejor que personas que han pasado por tu situación (E15).

En esta sociedad en la que cada uno va a lo suyo cuesta creer que haya un grupo de personas que pasan las tardes trabajando y pensando la mejor estrategia partiendo de la experiencia que ellos ya tienen (E14).

No podía imaginar encontrar aquí tanto apoyo, por mi situación estoy sola y me han hecho ver que, ante este problema no estoy sola (E12).

Los entrevistados han valorado de forma muy positiva el apoyo social recibido, sin su entorno todo habría sido más difícil, han jugado un papel clave en la superación de la situación adversa y han significado un pilar fundamental en el proceso en el que se han visto inmersos.

2.4. Visión de futuro como elemento facilitador Independientemente de las causas y consecuencias del proceso de desahucio, todas las personas entrevistadas reflexionan en torno a la reconstrucción de sus proyectos de vida.

Siempre se ha dicho que detrás de lo malo viene algo bueno, pero yo no quiero ni pensarlo, lo único que sé es que para lo que venga voy a estar preparada (E3).

Queda mucho por vivir aún, esta ha sido una etapa que una vez que termine tendremos que dejar atrás, sólo aprender para no volver a repetir los mismos errores (E14).

Los entrevistados prefieren vivir pensando en el presente, en ir recuperando poco a poco el equilibrio con el que vivían antes de verse afectados por el desahucio. Cuando se les pregunta sobre el futuro, confían en sus capacidades de superación y tratan de planear el camino a seguir, pero siempre paso a paso. Casi todos reconocen que han desarrollado fortalezas a nivel emocional y que han aprendido de la experiencia en la que se han visto inmersos o en la que en algunos casos continúan estando. Han aprendido a forjar el camino día a día.

No es que venga algo mejor, sino que yo voy a ser la encargada de buscarlo (E11).

$\mathrm{He}$ aprendido que si se quiere se sacan fuerzas de donde crees que ni las hay. Esto se lo estoy 
transmitiendo a mis hijos, les puede servir en el futuro ya que en la vida se enfrentarán a muchos problemas y quiero que se acuerden de nosotros y luchen como lo están haciendo su padre y su madre (E1).

Muchos aspiran a encontrar trabajo o mejorar las condiciones laborales que poseen en estos momentos, otros han decidido entregar su vivienda en dación en pago para lo que se están preparando psicológicamente - «Desprenderse de la vivienda no es fácil» (E6) - y otros han aceptado el proceso de desahucio debido a su inminente ejecución: «Ya es un hecho, en unos días abandono mi casa y me vuelvo a mi país» (E9).

Algunos de los entrevistados tienen la intención de seguir formando parte de la plataforma de afectados por las hipotecas ya que creen en la importante labor que lleva a cabo este movimiento social y están convencidos de que pueden servir de ayuda a otros en circunstancias similares.

\section{Conclusiones}

A partir de los resultados obtenidos se evidencia, como principal conclusión, la fuerza con la que la realidad de los desahucios está afectando a las personas que se ven implicadas en esta experiencia. La ejecución judicial forzosa que obliga a las familias al abandono de su vivienda, abre ante ellas un escenario de caos e inseguridad que les genera numerosas consecuencias de carácter personal, emocional y social, lo que de acuerdo con Navarro (2005) las sitúa en el inicio de un posible camino hacia la vulnerabilidad. Pero a su vez, también los resultados confirman, que es precisamente en esta experiencia de vulnerabilidad, donde las familias encuentran estructuras y recursos que les abren oportunidades de cambio $\mathrm{y}$ crecimiento ante esta adversidad.

En respuesta al objetivo del que parte este trabajo, los resultados muestran la presencia de factores internos y externos en las personas afectadas por la realidad de los desahucios que les permite activar respuestas resilientes ante la situación compleja en la que se encuentran. No se trata de personas invulnerables, como apuntaba Garmezy (1991), sino de quienes experimentan la dificultad, pero encuentran en la misma, una estructura de oportunidades para el crecimiento (Katzman, 1999).

Ante una situación adversa determinada, en este caso verse ante un desahucio, la persona «se rompe», llega a tocar fondo ya que es una situa- ción inesperada y desfavorable para cualquier ser humano. Sin embargo, tras tomar conciencia de ello, hay quienes son capaces de resurgir y encontrar en dicha situación, nuevas vías y oportunidades por las que avanzar. De acuerdo con Gauto de Paz (2007) el proceso de enfrentamiento a una adversidad es siempre un juego dinámico entre recursos, fortalezas y debilidades, que se desarrolla en cualquier momento y que necesita ser explicado para cada situación y persona concreta. Así pues, no es posible identificar un proceso único desde el que los participantes del estudio hayan conseguido superar y aprender ante la experiencia del desahucio, aunque si se ha podido comprobar cómo cada uno de ellos ha aprovechado sus fortalezas individuales - a veces no conocidas-, para construir una respuesta resiliente ante la situación amenazante (Mrazek y Mrazek, 1987): se han encargado de buscar información sobre el proceso de desahucio y las posibles soluciones a las que podían recurrir; han reconocido sus propios miedos y debilidades, compartiéndolos con otros; han sido capaces de tomar sus propias decisiones incluso pudiendo implicar éstas algún tipo de riesgo, creyendo siempre que toda nueva experiencia implica algún aprendizaje, etc.

Junto a los factores internos, la situación de adversidad que provoca un desahucio ha visibilizado el valor del capital social como estrategia resiliente (Katzman, 1999). Las relaciones interpersonales de protección, de apoyos comunitarios y familiares, los compromisos e implicación social que se adquieren con otros, etc. han sido elementos externos presentes en las respuestas resilientes que los participantes han ido construyendo (Coloma, 2009). Las fuentes de apoyo que han acompañado a los afectados por los desahucios han sido bastante heterogéneas, atendiendo a la composición familiar y a la red de apoyo social con la que cuenta cada persona (amigos, vecinos y conocidos) ofreciéndole apoyo emocional o material durante el proceso. Esta red de apoyo, se muestra como factor resiliente externo con el que las familias han contado en sus procesos de afrontamiento ante la experiencia del desahucio.

De forma especial, queda evidenciada en los resultados del presente estudio, la indiscutible labor de apoyo social que ejerce la Plataforma de Afectados por Hipoteca. El proceso de desahucio tiene un recorrido largo, en el que confluyen diversos factores que pueden hacer la travesía 
más simple o, complicarse hasta llegar a agotar física y mentalmente a todo aquel que se vea afectado de forma directa o indirecta. Más allá de esta función micro, hacia el individuo, la respuesta social solidaria surgida a través de estas plataformas, se dirige a su vez hacia las causas macro-estructurales que lo están provocando. Es en este doble plano, donde el enfoque de la resiliencia ofrece un paradigma inspirador para la intervención social, ayudando con ello a reenfo- car la mirada sobre las problemáticas sociales, las situaciones de riesgo, las políticas sociales o las estrategias de intervención (Gilgun, 1996). Dada la realidad emergente y preocupante que se plantea ante los desahucios, es de vital importancia continuar investigando en esta línea de cara a conocer cuáles son las estrategias utilizadas por las personas que se han visto afectadas por los mismos para tratar de diseñar una intervención adecuada.

\section{Referencias bibliográficas}

Arredondo, R. y Palma, M.O. (2013). Aproximación a la realidad de los desahucios. Perfil y características de las familias en proceso de desahucios en la ciudad de Málaga. Alternativas. Cuadernos de Trabajo Social, 20, 113-140. http://dx.doi.org/10.14198/ALTERN2013.20.07

Coloma, A. (2009). Una aproximación a la intervención del Trabajo Social comunitario en situaciones de catástrofes y desastres. Cuadernos de Trabajo Social, 22, 243-257.

Consejo General del Poder Judicial (2015). Informes periódicos. Efecto de la crisis en los órganos judiciales. Disponible en http://www.poderjudicial.es/cgpj/es/Temas/Estadistica_Judicial/Informes_e stadisticos/Informes_periodicos (Consultado el 13 de mayo de 2015).

Cortés, L. (2000). La vivienda como factor de exclusión social en la ciudad. Documentación Social, $119,295-312$

Escuela Pública de Salud de Andalucía. (2014). Estado de salud de la población afectada por proceso de desahucio. Stop Desahucios. Disponible en: https://granadastopdesahucios.files.wordpress. com/2014/10/resumen-ejecutivo-desahucios-final-240914b.pdf (Consultado el 3 de mayo de 2015).

Fundación FOESSA.(2013). La vivienda en España en el siglo XXI. Diagnóstico del modelo residencial y propuestas para otra política de vivienda. Disponible en: http://www.caritas.es/AdjuntoNoticiaDownload.aspx?Id=947 (Consultado el 7 de junio de 2015).

Gauto de Paz, G (2007). Resiliencia para reducir la vulnerabilidad a los riesgos de la vivienda pobre urbana. Cuadernos Geográficos, 46, 233-255.

Garmezy, N. (1991). Resiliency and vulnerability to adverse developmental outcomes associated with poverty. American Behavioral Scientist, 34(4), 416-430.

Gilgun, J. F. (1996). Human Development and Adversity In Ecological Perspective, Part 1: A Conceptual Framework en Families in Society: The Journal of Contemporary Social Services, 77(7), 395-402.

Kaztman, R. (coord.) (1999). Activos y Estructuras de oportunidades. Estudio sobre las raíces de la vulnerabilidad social en Uruguay. LC/MVD/R. 180. UN/CEPAL. Oficina de Montevideo. Uruguay.

Malagón, S. (2008). La vivienda y los procesos de exclusión social. Trabajo Social Hoy, Segundo Semestre, 101- 113.

Mrazek, P.J. y Mrazek, D. (1987). Resilience in children at high risk for psychological disorder. Journal of Pediatric Psychology, 12(3), 3-23.

Osborn, A. (1994). Resiliencia y estrategias de intervención. Ginebra: BICE.

Navarro, C. (2005). Indicadores de Vivienda y exclusión. Documentación Social, 138, 173-190.

Rutter, M. (1993). Resilience: Some conceptual considerations. Journal of Adolescent Health, 14, 626-631

Sabater, C. y Giró, J. (2015). La nueva pobreza. El desahucio como proceso de exclusión. Ehquidad International Welfare Policies and Social Work Journal, 3, 77-106. doi: 10.15257/ehquidad.2015.0004

Salgado, A.C. (2005). Métodos para medir la resiliencia: Una alternativa peruana. Liberalit, Revista de Psicología, 11, 41-48.

Terris, M. (1992). Tendencias actuales de la Salud Pública de las Américas. En: La crisis de la salud pública: reflexiones para el debate (pp. 185-204). Washington, D.C.: OPS, 540. 
Vanistendael, S. y Lecomte, J. (2002). La felicidad es posible. Despertar en niños maltratados la confianza en sí mismos: construir la resiliencia. Barcelona: Gedisa.

Valiño, V. (coord.) (2013). Emergencia habitacional en el Estado Español. Plataforma de Afectados por la Hipoteca (PAH) de Barcelona y Observatorio DESC. Recuperado de: http://afectadosporlahipoteca.com/wp-content/uploads/2013/12/2013-Emergencia-Habitacional_Estado_Espanyoldef.pdf (Consultado el 7 de junio de 2015). 